\title{
High Concordance between Trained Nurses and Gastroenterologists in Evaluating Recordings of Small Bowel Video Capsule Endoscopy (VCE)
}

\author{
Alessandra Guarini ${ }^{1}$, Francesca De Marinis ${ }^{1}$, Cesare Hassan $^{1}$, Raffaele Manta $^{2}$, Vincenzo De Francesco ${ }^{3}$, Bruno Annibale $^{4}$, \\ Angelo Zullo ${ }^{1}$
}

1) Gastroenterology and

Digestive Endoscopy, "Nuovo

Regina Margherita" Hospital,

Rome,

2) Digestive Endoscopy Unit,

"Civile S. Agostino-Estense"

Hospital, Baggiovara, Modena,

3) Gastroenterology Unit,

"Riuniti" Hospital, Foggia,

4) Department of Digestive and Liver Disease, Sant'Andrea

Hospital, "Sapienza"

University, Rome,

Italy

\begin{abstract}
Background \& Aims: The video capsule endoscopy (VCE) is an accurate and validated tool to investigate the entire small bowel mucosa, but VCE recordings interpretation by the gastroenterologist is time-consuming. A pre-reading of VCE recordings by an expert nurse could be accurate and cost saving. We assessed the concordance between nurses and gastroenterologists in detecting lesions on VCE examinations.

Methods: This was a prospective study enrolling consecutive patients who had undergone VCE in clinical practice. Two trained nurses and two expert gastroenterologists participated in the study. At VCE pre-reading the nurses selected any abnormalities, saved them as "thumbnails" and classified the detected lesions as a vascular abnormality, ulcerative lesion, polyp, tumor mass, and unclassified lesion. Then, the gastroenterologist evaluated and interpreted the selected lesions and, successively, reviewed the entire video for potential missed lesions. The time for VCE evaluation was recorded.

Results: A total of 95 VCE procedures performed on consecutive patients (M/F: 47/48; mean age: $63 \pm 12$ years, range: $27-86$ years) were evaluated. Overall, the nurses detected at least one lesion in 54 (56.8\%) patients. There was total agreement between nurses and gastroenterologists, no missing lesions being discovered at a second look of the entire VCE recording by the physician. The pre-reading procedure by nurse allowed a time reduction of medical evaluation from 49 (33-69) to 10 (8-16) minutes (difference: -79.6\%).

Conclusions: Our data suggest that trained nurses can accurately identify and select relevant lesions in thumbnails that subsequently were faster reviewed by the gastroenterologist for a final diagnosis. This could significantly reduce the cost of VCE procedure.
\end{abstract}

Key words: video capsule endoscopy - nurse - concordance - cost.

Abbreviations: GI: gastrointestinal; OGIB: obscure gastrointestinal bleeding; VCE: video capsule endoscopy.

\section{INTRODUCTION}

Video capsule endoscopy (VCE) is a non-invasive and validated technique which allows entire small bowel mucosa visualization [1]. According to the current European guidelines, VCE is recommended for patients with obscure gastrointestinal bleeding (OGIB), suspected Crohn's disease with negative ileocolonoscopy, suspected small-bowel tumours, and inherited polyposis syndromes [1]. Other potential indications are unexplained chronic diarrhoea, drug-related enteropathy, and some forms of coeliac disease [1-3]. The major limitation of $\mathrm{VCE}$ is the time required to review the recording, which may range from 60 to 120 minutes [4]. This is a time-consuming procedure for a gastroenterologist. As the VCE is an automatic registration, the reading operator does not affect the quality of stored images, but he/she is required to search the lesions and to interpret them. In this context, it is expected that an expert gastroenterology nurse, specifically trained on VCE procedure, would be able to accurately detect the main small bowel lesions [5]. Indeed, there is some evidence that the pre-reading of VCE recordings by expert nurses is accurate, permitting a time reduction for the physician's evaluation without losing relevant lesions [6]. In a previous pilot study [7], we assessed the ability of two trained nurses in detecting the small bowel lesions in comparison with two expert gastroenterologists; the nurse evaluation was highly (95.6\%) accurate in detecting small bowel lesions, with a 100\% concordance with the gastroenterologist. 
In this study, we aimed to validate these results in a large, prospective study on VCE procedures consecutively performed in clinical practice.

\section{METHODS}

\section{VCE procedure}

All consecutive patients who underwent small bowel examination with VCE in routine clinical practice in a single centre were enrolled in this study. Before the procedure, all patients underwent clinical evaluation by a gastroenterologist to ascertain the indication, to rule out the contraindications, and to obtain a written informed consent. Then, a nurse gave all information regarding bowel preparation and the entire VCE procedure. The bowel preparation consisted of polyethylene glycol solution (2 litters with two sachets of polyethylene glycol 1000) ingested between 7 p.m. and 9 p.m. the day before the examination. The patients remained fasting overnight. At morning, the nurse applied the sensor arrays, administered the capsule, and verified that it reached the stomach. The patient was discharged and he/she was allowed to drink clear liquids 2 hours after ingestion of the capsule and then eat a light snack after a further 2 hours. The nurse also instructed the patient in correctly disconnecting the recorder pack after 8 hours. The successive morning, the nurse downloaded the video to a dedicated workstation. The PillCam Small Bowel (Medtronic, Milan, Italy) was used.

\section{VCE evaluation}

Two trained nurses and two expert gastroenterologists, who routinely perform VCE studies in clinical practice, participated in the study. In detail, the two nurses with more than 20 years experience on upper and lower gastrointestinal (GI) endoscopy, attended a specific, intensive 2-day training International course, held in the Catholic University of Rome in 2011, where they learned to identify small bowel lesions on more than $50 \mathrm{VCE}$ recordings. Thereafter, they routinely performed VCE pre-reading, before the gastroenterologist's evaluation, incurring more than 7 years' experience on this procedure [7]. They randomly evaluated the VCE recordings and selected the first image of stomach, duodenum, and colon. The nurse saved all the endoscopic lesions or abnormalities that were saved as "thumbnails". The nurse also classified the lesions according to the following categories: a) vascular lesion (angiodysplasia/lymphangiectasia); b) ulcerative (erosions/ ulcers) finding, c) polyp, d) tumour masses, and e) unclassified lesion, irrespective of the number, when lesions were similar (i.e. multiple erosions, angiodyplasias, etc). When different lesions were detected, the patient was classified according to the most clinically relevant lesion. For each lesion, signs of active (fluid blood) or recent bleeding (clots) were recorded. Successively, the two gastroenterologists randomly and independently evaluated and interpreted the lesions selected by the nurse as "thumbnails", also briefly reviewing the video related to the selected lesion (quick medical view). Thereafter, the involved gastroenterologist reviewed the entire video to search for potential missed lesions. Therefore, one nurse and one gastroenterologist reviewed the entire VCE recording. The total time for each VCE evaluation by the nurses and gastroenterologists (quick medical view and entire medical view) were recorded.

\section{RESULTS}

The VCE recordings of 95 consecutive patients (M/F: 47/48; mean age: $63 \pm 12$ years, range: $27-86$ years) were evaluated. Indications for VCE included OGIB $(\mathrm{n}=76)$, suspected Crohn's disease $(n=6)$, hereditary polyposis syndrome (Peutz-Jeghers: $\mathrm{n}=3$; Familial Adenomatous Polyposis: $\mathrm{n}=5$ ), and chronic diarrhoea $(n=4)$, suspected neuroendocrine tumor $(n=1)$. The entire small bowel was visualized in all but one patient, in whom the capsule failed to overcome a stenotic, neoplastic lesion of the jejunal tract within the 8 hours of registration. However, the device was spontaneously expulsed the day after, and no case of retained VCE was observed. Overall, the nurses described 41 negative VCE (without any pathological finding), whilst the presence of at least one lesion in the remaining 54 (56.8\%) patients. In detail, there were 17 cases with vascular lesions (angiodysplasia or lymphangiectasia), 20 with ulcerative lesions (ulcer or erosion), 11 with polypoid lesions, 5 with tumoral masses, and one unclassified lesion. Active bleeding was recorded in 4 patients and recent bleeding in 1 case. As shown in Table I, there was a total agreement between nurses and physicians concerning the bowel preparation as well as the detection of small bowel lesions. Indeed, the second examination of the entire VCE recording by the physician failed to find other relevant mucosal abnormalities. The overall reading time (median; range) was 58 (45-79) minutes for nurses, 10 (8-16) and 49 (33-69) minutes for the quick and entire medical view by the gastroenterologists.

Table I. Concordance between nurses and gastroenterologists in finding small bowel lesions at videocapsule endoscopy

\begin{tabular}{lccc}
\hline Variable & Nurse & Gastroenterologist & P value \\
\hline Bowel preparation & & & 0.8 \\
\hline Excellent & 65 & 67 & \\
Good & 25 & 23 & \\
Fair & 4 & 4 & 1 \\
Poor & 1 & 1 & \\
\hline Lesions detected & & & \\
\hline No abnormality & 41 & 41 & \\
Angiodysplasia/ & 17 & 17 & \\
Lymphangiectasia & & & \\
Ulcer/erosion & 20 & 20 & \\
Polyp & 11 & 5 & \\
Tumoral mass & 5 & $4 / 1$ & \\
Unclassified & 1 & & \\
Bleeding (active/recent) & $4 / 1$ & & \\
\hline
\end{tabular}

Therefore, the pre-reading procedure by the nurse allowed a time reduction of the medical evaluation by $79.6 \%$. The final diagnosis according to the gastroenterologist's evaluation in the differently categories are reported in Table II. Pictures of some detected lesions were provided in Fig. 1. 
Table II. Final endoscopic diagnosis performed by gastroenterologist.

\begin{tabular}{lcl}
\hline Lesions detected & Nurse & Gastroenterologist \\
\hline $\begin{array}{l}\text { Angiodysplasia/ } \\
\text { Lymphangiectasia }\end{array}$ & 17 & Angiodysplasia/Lymphangiectasia: 17 \\
Ulcer/erosion & 20 & $\begin{array}{l}\text { Erosions: } 14 \\
\text { Crohn's diseases: } 5 \\
\text { Meckel's diverticulum: } 1\end{array}$ \\
Polyp & & Polyp: 11 \\
Tumoral mass & 5 & $\begin{array}{l}\text { Tumor: } 4^{*} \\
\text { Melanoma: } 1\end{array}$ \\
Unclassified & 1 & Foreign body: $1^{* *}$ \\
\hline
\end{tabular}

${ }^{\star}$ Pathological diagnosis: Adenocarcinoma $(\mathrm{n}=2)$; NET $(\mathrm{n}=1)$; GIST $(\mathrm{n}$ $=1) ;{ }^{*} \mathrm{~A}$ fish bone removed at endoscopy.

\section{DISCUSSION}

The VCE is increasingly performed in clinical practice, mainly to ascertain the causes of OGIB. In these patients, the detection rate of VCE was evaluated at $60.5 \%$ (95\% CI 57.2-63.9) in a large meta-analysis [8]. Moreover, the role of VCE is clinically relevant even following normal findings, as the rebleeding rate in these patients is distinctly low (5.6-16\%) [9-11].

The main relevant limitation of VCE is the long time needed to review the recordings. The evaluation of small bowel mucosa is performed by an expert endoscopist who dedicates a slot of one working day for reading the entire VCE recording, with relevant costs [12]. Therefore, a pre-reading procedure performed by an expert and a specifically trained nurse would result in a time reduction of successive medical evaluations, as long as the initial assessment was accurate. However, only a few studies are available in this setting. It has been found that the accuracy of trained nurses in interpreting VCE features, particularly for relevant lesions, is as high as $93 \%-100 \%$ [7, 13-15]. To our knowledge, this is the largest study assessing the accuracy of nurse pre-reading VCE recordings, following another cohort that included 102 patients [16]. Our data demonstrated that trained nurses were able to detect and correctly classify small bowel lesions, and no relevant lesions were missed during the nurse pre-reading, as confirmed by the successive medical evaluation. Of note, while the reading time for the entire VCE recording did not differ between nurses and gastroenterologists, the pre-reading performed by the nurses allowed an important time reduction for the following medical evaluation from a median of 49 to only 10 minutes, corresponding to a $80 \%$ reduction from the total time. This finding is consistent with the results of a recent study where nurse pre-reading was associated to a $73 \%$ reduction time (from 34 to 9 minutes) of the successive medical evaluation [14]. Therefore, a pre-reading VCE by trained nurse followed by short medical evaluation of selected abnormalities would appear highly accurate and would lead to a significant cost saving. Indeed, it has been calculated that a preliminary review by the nurse distinctly reduces the cost for each examination by $30 \%$ in Italy and by $43 \%$ in Israel $[6,14]$. The reading

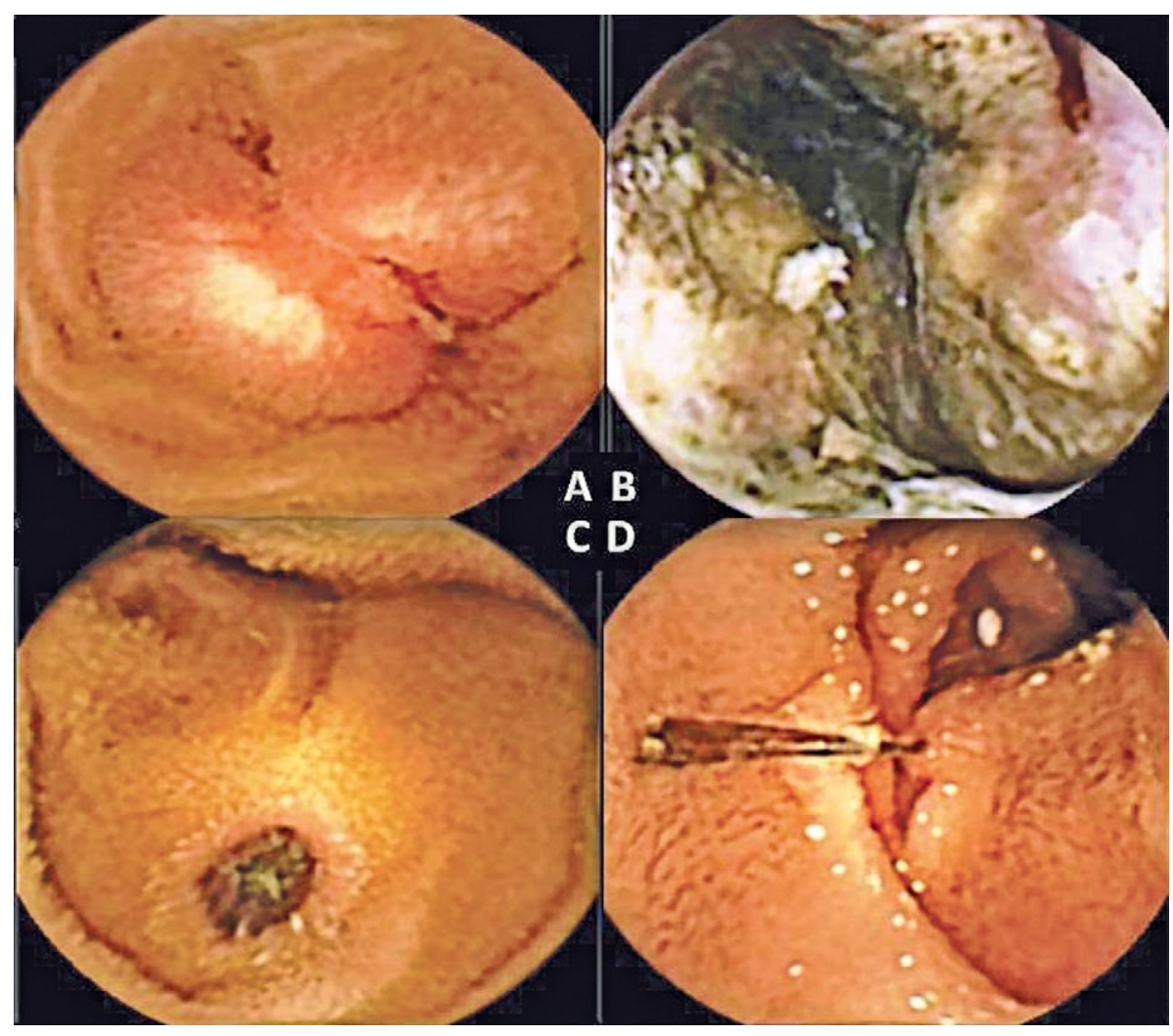

Fig. 1. (A) Sub-stenotic mass corresponding to a neuroendocrine tumor at post-surgical evaluation. (B) Polypoid mass corresponding to lymphangioma at post-surgical evaluation. (C) Metastatic, ulcerated melanoma. (D) A fixed fish bone in the third part of duodenum removed at endoscopy. 
time could be further shortened with the implementation of dedicated software, designed to recognize polyps or Crohn's disease lesions $[17,18]$.

When could a nurse expert in digestive endoscopic examinations be considered "trained" in VCE? Unfortunately, only few data exist on the learning curve for VCE interpretation. A study calculated that more than 20 examinations were needed for gastroenterology fellows before assessing competence [19], and more than 40 examinations were found to be sufficient for a nurse expert in digestive endoscopic examinations [20,21]. A previous large experience in digestive endoscopy was found to increase the detection rate by nurses to $89 \%$ [22]. The nurses who participated in our study were specifically trained in recognizing the most common lesions of the small bowel at VCE during a dedicated course. Moreover, they continued training with gastroenterologist supervision in routine practice during 7 years. In our previous pilot study on 46 patients, the nurses' accuracy was as high as $95.6 \%$ when compared to the gastroenterologists' evaluation [7], and data of the present study confirmed this result. A recent systematic review of 11 studies involving 13 nurses calculated an accuracy rate of $86.2 \%$ (range: $75-94.5 \%$ ) in detecting the overall lesions, despite the fact that 7 of them had not attended a specific course for training, and 3 were trained with only 5 demo videos and 10 supervised VCE readings [22]. Our data suggest that training with at least 50 evaluations is required for achieving a very high accuracy in VCE pre-reading by nurses. Of note, some studies found that an expert nurse (60 VCE procedures) was equally accurate as the expert physician (250 VCE procedures) and more accurate than physicians with $100 \mathrm{VCE}$ procedures experience, irrespective of the software used $[23,24]$.

Although currently limited to VCE pre-reading, the potential role of nurse for an independent selection of pictures, their interpretation and the realization of a final report has been also evaluated in a recent study, but the accuracy of reports was $80 \%$ [15]. Therefore, to date, the trained nurses' reading is accurate for finding and selecting lesions, which should be interpreted by the gastroenterologists in the clinical context, for the official report. Indeed, it has been already affirmed that "In endoscopy, as in life, detection skills are related to attentiveness and awareness. It is in the interpretation of findings that expertise comes into play" [25].

\section{CONCLUSION}

Our data suggest that a trained nurse may accurately evaluate VCE findings, select the thumbnails of all mucosal irregularities which subsequently may be faster reviewed by the gastroenterologist for a final diagnosis. This would significantly reduce the cost of VCE procedure.

\section{Conflicts of interest: None to declare.}

Authors' contributions: A.G. and A.Z. conceived, designed, analyzed data, and wrote the manuscript. A.G., F.D.M., C.H. and A.Z. performed VCE evaluations. R.M. and B.A. reviewed the manuscript with constructive criticisms.

\section{REFERENCES}

1. Pennazio M, Spada C, Eliakim R, et al. Small-bowel capsule endoscopy and device-assisted enteroscopy for diagnosis and treatment of small-bowel disorders: European Society of Gastrointestinal Endoscopy (ESGE) Clinical Guideline. Endoscopy 2015;47:352-376. doi:10.1055/s-0034-1391855

2. Zullo A, De Francesco V, Manta R, Ridola L, Lorenzetti R. A challenging diagnosis of jejunal adenocarcinoma in a celiac patient: case report and systematic review of the literature. J Gastrointestin Liver Dis 2017;26:411-415. doi:10.15403/jgld.2014.1121.264.zet

3. Furnari M, Buda A, Delconte G, et al. The role of wireless capsule endoscopy (WCE) in the detection of occult primary neuroendocrine tumors. J Gastrointestin Liver Dis 2017;26:151-156. doi:10.15403/ jgld.2014.1121.262.wce

4. Costamagna G, Shah SK, Riccioni ME, et al. A prospective trial comparing small bowel radiographs and video capsule endoscopy for suspected small bowel disease. Gastroenterology 2002;123:999-1005. doi:10.1053/gast.2002.35988

5. Sidhu R, Sakellariou P, McAlindon ME, et al. Is formal training necessary for capsule endoscopy? The largest gastroenterology trainee study with controls. Dig Liver Dis 2008;40:298-302. doi:10.1016/j.dld.2007.11.022

6. Bossa F, Cocomazzi G, Valvano MR, Andriulli A, Annese V. Detection of abnormal lesions recorded by capsule endoscopy. A prospective study comparing endoscopist's and nurse's accuracy. Dig Liver Dis 2006;38:599-602. doi:10.1016/j.dld.2006.03.019

7. Guarini A, De Marinis F, Hassan C, Spada C, Bruzzese V, Zullo A. Accuracy of trained nurses in finding small bowel lesions at video capsule endoscopy. Gastroenterol Nurs 2015;38:107-110. doi:10.1097/ SGA.0000000000000096

8. Liao Z, Gao R, Xu C, Li ZS. Indications and detection, completion, and retention rates of small-bowel capsule endoscopy: a systematic review. Gastrointest Endosc 2010;71:280-286. doi:10.1016/j.gie.2009.09.031

9. Lai LH, Wong GL, Chow DK, Lau JY, Sung JJ, Leung WK. Long-term follow-up of patients with obscure gastrointestinal bleeding after negative capsule endoscopy. Am J Gastroenterol 2006;101:1224-1228. doi:10.1111/j.1572-0241.2006.00565.x

10. Ribeiro I, Pinho R, Rodrigues A, et al. What is the long-term outcome of a negative capsule endoscopy in patients with obscure gastrointestinal bleeding? Rev Esp Enferm Dig 2015;107:753-758.

11. Tziatzios G, Gkolfakis P, Hassan C, et al. Meta-analysis shows similar re-bleeding rates among Western and Eastern populations after index video capsule endoscopy. Dig Liver Dis 2018;50:226-239. doi:10.1016/j. dld.2017.12.031

12. Rondonotti E, Soncini M, Girelli C, et al; AIGO, SIED, SIGE Lombardia. Cost estimation of small bowel capsule endoscopy based on "real world" data: inpatient or outpatient procedure? Dig Liver Dis 2010;42:798-802. doi:10.1016/j.dld.2010.03.006

13. Levinthal GN, Burke CA, Santisi JM. The accuracy of an endoscopy nurse in interpreting capsule endoscopy. Am J Gastroenterol 2003;98:2669-2671. doi:10.1111/j.1572-0241.2003.08726.x

14. Niv Y, Niv G. Capsule endoscopy examination - preliminary review by a nurse. Dig Dis Sci 2005;50:2121-2124. doi:10.1007/s10620-005-3017-7

15. Dreanic J, Barret M, Dhooge M, et al. Small bowel capsule endoscopy: may we delegate it to nurses? Clin Res Hepatol Gastroenterol 2018;42:168-173. doi:10.1016/j.clinre.2017.10.008

16. Dokoutsidou H, Karagiannis S, Giannakoulopoulou E, et al. A study comparing an endoscopy nurse and an endoscopy physician in capsule 
endoscopy interpretation. Eur J Gastroenterol Hepatol 2011;23:166-170. doi:10.1097/MEG.0b013e3283433abf

17. Charisis VS, Hadjileontiadis LJ. Potential of hybrid adaptive filtering in inflammatory lesion detection from capsule endoscopy images. World J Gastroenterol 2016;22:8641-8657. doi:10.3748/wjg.v22.i39.8641

18. Yuan Y, Meng MQ. Deep learning for polyp recognition in wireless capsule endoscopy images. Med Phys 2017;44:1379-1389. doi:10.1002/mp.12147

19. Rajan E, Iyer PG, Oxentenko AS, et al. Training in small-bowel capsule endoscopy: assessing and defining competency. Gastrointest Endosc 2013;78:617-622. doi:10.1016/j.gie.2013.05.010

20. Brock AS, Freeman J, Roberts J, Dantzler TE, Hoffman BJ. Resource-efficient tool for training novices in wireless capsule endoscopy. Gastroenterol Nurs 2012;35:317-321. doi:10.1097/ SGA.0b013e3182604d2f

21. Sidhu R, Sanders DS, Kapur K, Marshall L, Hurlstone DP, McAlindon ME. Capsule endoscopy: is there a role for nurses as physician extenders? Gastroenterol Nurs 2007;30:45-48.
22. Yung DE, Fernandez-Urien I, Douglas S, et al. Systematic review and meta-analysis of the performance of nurses in small bowel capsule endoscopy reading. United European Gastroenterol J 2017;5:1061-1072. doi:10.1177/2050640616687232

23. Shiotani A, Honda K, Kawakami M, et al. Evaluation of RAPID 5 Access software for examination of capsule endoscopies and reading of the capsule by an endoscopy nurse. J Gastroenterol 2011;46:138-142. doi:10.1007/s00535-010-0312-7

24. Shiotani A, Honda K, Kawakami M, et al. Analysis of small-bowel capsule endoscopy reading by using Quickview mode: training assistants for reading may produce a high diagnostic yield and save time for physicians. J Clin Gastroenterol 2012;46:e92-e95. doi:10.1097/ MCG.0b013e31824fff94

25. Koulaouzidis A, Toth E. Optimizing the interpretation of capsule endoscopic images: shortsighted or taking the long view? Dig Dis Sci 2015;60:1519-1521. doi:10.1007/s10620-015-3601-4 